\title{
The Principle Underlying Quantum Mechanics
}

\author{
Aage Bohr, ${ }^{1}$ Ben R. Mottelson, ${ }^{2,3}$ and Ole Ulfbeck ${ }^{1}$
}

Received May 22, 2003; revised October 2, 2003

\begin{abstract}
The present article reports on the finding of the principle behind quantum mechanics. The principle, referred to as genuine fortuitousness, implies that the basic event, a click in a counter, comes without any cause and thus as a discontinuity in spacetime. From this principle, the formalism of quantum mechanics emerges with a radically new content, no longer dealing with things (atoms, particles, or fields) to be measured. Instead, quantum mechanics is recognized as the theory of distributions of uncaused clicks that form patterns laid down by spacetime symmetry and is thereby revealed as a subject of unexpected simplicity and beauty. The departure from usual quantum mechanics is strikingly borne out by the absence of Planck's constant from the theory. The elimination of indeterminate particles as cause for the clicks, which the principle of genuine fortuitousness implies, is analogous to the elimination of the ether implied by the principle of relativity.
\end{abstract}

KEY WORDS: foundation of quantum mechanics; fortuitousness; discontinuity.

\section{INTRODUCTION}

Quantum mechanics, in contrast to relativity theory, lacks a basic principle, from which the theory develops in a compelling manner. In fact, the quantum mechanical formalism was discovered by ingenious guesswork which was given an interpretation in terms of probabilities for the results of measurements. This interpretation involves the notion that there exists particles as mediators in producing the basic event, a click in a counter.

\footnotetext{
${ }^{1}$ The Niels Bohr Institute, University of Copenhagen, DK-2100, Copenhagen, Denmark.

${ }^{2}$ Nordita, DK-2100, Copenhagen, Denmark.

${ }^{3}$ To whom correspondence should be addressed; e-mail: mottelson@nbi.dk
} 
The particle is, thereby, seen as the cause for the click. Yet it is maintained that the click is fortuitous. ${ }^{4}$

It is the purpose of the present article to show that the acceptance of a complete break with causality, by which the individual click is recognized as entirely uncaused, provides a missing element in the logical structure of quantum mechanics. Indeed, this conception will be seen to constitute the principle underlying quantum mechanics that has hitherto been lacking. It is found to have farreaching consequences for the content of the theory.

The emphasis of the article is on the simple and compelling directness by which the principle enforces a new conceptual structure of quantum mechanics. This simplicity makes possible a presentation that exploits the focus implied by brevity and, at the same time, is self-contained. It has been a special challenge that the principle goes against deeply held beliefs, and we hope that the reader may to begin with suspend his disbelief in order that the simplicity and logical coherence of the resulting structure may be comprehended. ${ }^{5}$

\section{UNCAUSED CLICK. DISCONTINUITY}

A click entirely without a cause, and thus coming by itself, has the novel property of an onset, a beginning from which it develops. The onset, having no precursor, comes as a discontinuity in space and time and is, therefore, unanalyzable. The sharpness with which the discontinuity can be established is limited only by the resolution of the counter. The notion of a discontinuity was invoked already in the early quantum theory to characterize a complete transition between stationary states in an atom. However, the discontinuity is now recognized as a macroscopic event, which takes place in the counter, and not something happening in an atom.

\footnotetext{
${ }^{4}$ In early quantum theory (1913-1925), it was recognized that the description of individual atomic events involves statistical elements. The creation of quantum mechanics (1925-1927) provided the formalism for evaluating the probabilities. However, the lack of a basic principle underlying the theory leaves unanswered the question of how and where the break with causality takes place and has led to a continuing discussion of these issues. An extensive literature is reprinted in the book Quantum Theory and Measurement (Ref. 2). The discussion has been further expanded in the last decades, see, for example, Refs. 3, 4, and references contained therein.

${ }^{5}$ The concept of genuine fortuitousness was introduced in Ref. 1 in the context of an analysis of matrix variables in usual quantum mechanics. The more farreaching content of genuine fortuitousness was not recognized at the time of writing Ref. 1. Thus, the principle, which supplies the missing element in the logics, and from which quantum mechanics develops as the embodiment of spacetime symmetry, remained beyond the horizon.
} 
The characterization of fortuitousness in terms of a click that comes by itself, as a discontinuity, is quite distinct from the content that has been given to fortuitousness in usual quantum mechanics. In order to differentiate, an event that comes entirely without a cause as a discontinuity will be referred to as genuinely fortuitous. Absence of cause and discontinuity are thus inseparable characteristics of the genuinely fortuitous click, ${ }^{6}$ and in the following when one is used the other is implied. Genuine fortuitousness with these novel feature becomes the central new concept in the theory, replacing quantum (= particle).

The individual click with its discontinuity is entirely beyond law. However, patterns defining probabilities may emerge, when the clicks are characterized by only a few numbers (clicks in low resolution), whose significance will appear in the following. Whether a click belongs to a postulated distribution is a matter for the experimenter and, in the last resort, for the physics community to decide upon. These probability distributions exhaust the content of the theory of uncaused clicks.

The clicks, of course, do not occur in pure emptiness, but in the context of counters and apparatus, which provide a classical background for the exploration of the clicks. While the click violates the causality of classical laws, it is itself an event that takes place in space and time, the framework for ordering experience.

\section{DERIVATION OF QUANTUM MECHANICS FROM GENUINE FORTUITOUSNESS}

It might seem that genuine fortuitousness cannot lead to a rational theory. Surprisingly, however, it turns out to be very powerful in its implications.

First, a click that comes by itself as a discontinuity is not connected to a thing (object) that existed previously and thus cannot be a measurement of any thing. Hence, the complete break with causality leads to a theory having the higly novel character of no longer involving measurement.

Second, since particles are excluded as intermediaries, only geometry is left on the stage, and the very symmetry of spacetime provides uniquely the mathematical framework for the click distributions.

${ }^{6}$ Unease about whether the notion of fortuitousness has been properly grasped in quantum mechanics has been expressed from many sides. However, to our knowledge, the consequences of genuine fortuitousness have not been confronted. 


\subsection{Assignment of Proper Value to Click}

The symmetry of spacetime (relativistic invariance) encompasses the invariance of four-dimensional spacetime under the group of translations, rotations, and reflections. These coordinate transformations are the group elements, each of which has a set of proper values, such as \pm 1 for reflections, and a wave number giving the proper value of a translation. Rotations have fixpoints in spacetime that are left invariant under the rotation and thereby single out points in spacetime. A Lorentz transformation as a spacetime rotation singles out a point in space, for a specified time. This link between space and time, through relativity, is crucial for the theory to be developed, even in the non-relativistic corner of spacetime invariance; see footnote 5 . The coordinate transformations do not all commute, and the spacetime invariance group is, therefore, non-Abelian (Poincaré symmetry).

The connection between click and symmetry is established by assigning a proper value of one or more symmetry elements to the click. This assignment is based on the set-up constructed by the experimenter for this purpose. Thus, reflection symmetry can be assigned by means of a partially reflecting mirror, and translation symmetry (wave number) by a diffraction spectrometer. The location of a spot on a photographic plate can be related to the proper value of a Lorentz transformation, which is unity for a transformation leaving the location invariant. (The ultimate test of the assignment of proper values to the clicks involves the theory itself, as described in Sec. 3.3).

\subsection{Embodiment of Spacetime Symmetry}

The question of how spacetime symmetry impresses itself on the distribution of proper values turns out to have a definite answer, by which the distributions appear as an embodiment of symmetry. The present section aims at conveying the logical connections, while the mathematics is spelled out in the Appendix. In the embodiment, the mathematical constraints that express the interweaving of the symmetry elements in the non-Abelian group are transferred into the same set of constraints imposed on the click distributions. $^{7}$

\footnotetext{
${ }^{7}$ The unique manner, in which a representation of symmetry impresses itself on the distribution of proper values was exhibited in Ref. 5, in which the elements of spacetime invariance were seen as the basic variables of quantum mechanics ("primary manifestation of symmetry"). At the time of the writing, genuine fortuitousness was not yet in sight, and symmetry was, therefore, not yet liberated from association with particles (quanta). Conceptual problems that go with indeterminacy and measurement of variables, thus, remained. In the theory of causeless clicks, the embodiment of symmetry itself provides the organizing principle without any reference to variables.
} 
These constraints are found to be of such a character that the distributions of proper values can be encoded in a vector, the click vector, in symmetry space (Hilbert space). Thus, the click vector has components (a wave function) that yield the probability distributions of clicks, which have been assigned proper values for a particular symmetry element. The encoding in the click vector implies that, for symmetry elements that do not commute, the distributions obey reciprocal constraints (uncertainty relations).

It is emphasized that the emergence of probabilities that can be derived from a wavefunction (probability amplitude) is not a matter of interpreting a formalism but is a deduction from the embodiment of nonAbelian spacetime symmetry in distributions of uncaused clicks. In turn this deduction constitutes the implementation of genuine fortuitousness.

The usual role of symmetry in physics has been to provide tools for analyzing the behaviour of objects, including continuous media, and this role of spacetime symmetry is powerful in handling the formalism of usual quantum mechanics. Quite a different role is displayed by symmetry through its embodiment in the click distributions. There are here no objects to be characterized by the tools of symmetry, and it is the embodiment of symmetry itself that constitutes the theory.

\subsection{Time Evolution}

The time evolution of the symmetry elements is part of the group structure and implies a continuous evolution of the click distributions described by a differential equation in $\operatorname{time}^{8}$ (the Schrödinger equation). The time evolution of the click vector is affected by what is in spacetime, such as electric and magnetic fields that are part of the apparatus. The presence of such external agents is incorporated into the theory by terms in the equations for the time development of the click vector. The time development caused by the electromagnetic fields involves a coupling constant (electric charge), which is a dimensionless quantity; see Sec. 5 and footnote 6 .

In the manner described, the theory accounts for the effects of the apparatus on the click distributions. This application of the theory provides the test that the proper values have been consistently assigned to the clicks.

\footnotetext{
${ }^{8}$ Causal behaviour comes about because the link between space and time implies that the commutation between a spatial translation and a Lorentz transformation is a time displacement. Thus, the non-relativistic Schrödinger equation hinges on the relativistic link between space and time. This point is discussed in more detail in Ref. 5, Sec. IV D; see also Ref. 6, where relativity is recognized as basic for the canonical commutator between position and momentum.
} 


\subsection{Correlations}

The discontinuity of the onset implies that clicks belonging to different onsets are totally uncorrelated. However, the discontinuity can occur at two or more separate locations, giving rise to clicks which come in coincidence forming a single genuinely fortuitous event. The occurrence of such multiplets of clicks opens the possibility for correlations in proper values as well as in the onset-times within the multiplet. The discontinuity can also occur at locations with time-like separations.

While the uncorrelated clicks are described by irreducible representations (see Appendix), correlations are encoded in a click vector in terms of a superposition of product vectors involving as many irreducible representations as the number of clicks belonging to the discontinuity. The correlated click distributions define dimensionless couplings, which can be determined from an analysis of the time development of multiplets of clicks.

\subsection{Principle Underlying Quantum Mechanics}

In summary, genuine fortuitousness by which clicks come without a cause, in discontinuities, is seen to provide the rationale for the formalism of quantum mechanics. In fact, from this basis, the formalism emerges in a compelling manner as the embodiment of spacetime symmetry and, hence, with an unexpected simplicity and beauty. With the new logical structure, dictated by genuine fortuitousness itself, the theory has a physical content differing radically from that of usual quantum mechanics.

The conceptual revolution brought about by relativity theory was based on a clear principle provided by the invariance of spacetime. In constrast, quantum mechanics was created by the discovery of the appropriate equations in the absence of a principle that could determine the meaning of these equations. It can now be seen that the lacking principle underlying quantum mechanics is genuine fortuitousness in a spacetime governed by relativistic invariance.

\section{THE DOWNWARD PATH. ATOMS AS PHANTASMS}

It is a hallmark of the theory based on genuine fortuitousness that it does not admit physical variables. It is, therefore, of a novel kind that does not deal with things (objects in space), or measurements, and may be referred to as the theory of no things. 
While in this theory there are no electrons or atoms as things that could produce an event, the embodiment of symmetry involves symbolic structures which may be termed electron wave functions, proton wave functions, electron fields, proton fields, etc. These symbolic structures have quantum numbers that in usual quantum mechanics have been associated with particles.

The arguments of the wave functions, in the theory of no things, are not values of variables, as in usual quantum mechanics, but proper values of elements of spacetime symmetry. Thus, the argument may be a space coordinate $x$ related to a proper value of a Lorentz transformation, but the coordinate is not a position of a thing. Similarly, an atomic wave function is a solution of the wave equation, depending on two, or more, space coordinates that are not positions of things. Indeed, atoms and particles as things are phantasms (things imagined). The downward path from things stops with the uncaused click.

The point is illustrated by a cloud chamber picture showing a multiplet of correlated clicks (droplets) belonging to a single discontinuity occurring at the locations of the clicks. (This multiplet of clicks with timelike separations is analogous to multiplets with space-like separations considered in Sec. 6.4, which also arise from a single discontinuity). The droplets in the cloud chamber form a track that vividly conveys the image of a particle passing through the chamber, but this imagined thing is a phantasm. There are no things beyond the droplets.

In an unexpected manner, the theory of no things completes the elimination of the concept of motion in space and time. This development was initiated by the introduction (1913) of the notion of a stationary state.

\section{ABSENCE OF PLANCK'S CONSTANT. THE DIMENSION OF MASS}

In the theory of uncaused clicks, the formalism of quantum mechanics emerges as the embodiment of spacetime symmetry, without invoking Planck's constant. Indeed, this constant has no place in the theory, since the representation of spacetime symmetry involves only quantities with the dimensions of space and time.

External agents, such as electric and magnetic fields, enter into the equations for the click vector, as mentioned above, and are thereby expressed in dimensions involving only space and time, together with 
dimensionless coupling constants. ${ }^{9}$ In this manner, it is seen that also the quantities of classical physics acquire dimensions exclusively involving space and time.

The issue concerning Planck's constant goes to the core of the question of what are the basic dimensions of physics. In usual quantum mechanics, as in classical mechanics, mass is a quality with its own dimension beyond those of space and time. In the theory of no things, the dimension of mass is absent, since spacetime symmetry involves only length and time. The theory, thereby, achieves a reduction in the number of dimensions to those of spacetime itself which is, of course, unrelated to the choice of units. The elimination from physics of the concept of mass as an independent dimension provides a hitherto unrecognized insight revealed by genuine fortuitousness.

\section{THE THEORY OF CAUSELESS CLICKS AND USUAL QUANTUM MECHANICS}

The theory of causeless clicks as presented above builds upon a simple principle. On this background, usual quantum mechanics appears as an attempt to express the mathematical results of the formalism in terms of generalized classical concepts involving Planck's constant. Thereby it was taken for granted that the notion of particles as things causing the clicks carries over from the classical imagery.

It can now be seen that this violation of the principle is responsible for the conceptual problems that have remained issues in the debate on the understanding of quantum mechanics (indeterminacy, fortuitousness, completeness, measurement, state of object, correlation, particle-wave duality). Genuine fortuitousness removes these conceptual problems, as will be discussed in the following.

\subsection{Indeterminacy}

In usual quantum mechanics, the particle is a thing that is observed by the click it produces. At the same time, the notion of a particle with indeterminate variables implies limitations for visualizing the particle as a thing. The resulting framework admits statements like "The particle passes

\footnotetext{
${ }^{9}$ For example, an analysis of the clicks registering the spectral lines of the hydrogen atom with inclusion of the fine structure allows the determination of the dimensioneless elementary charge as well as the length scale of the atom. On this basis, external electric and magnetic fields can be obtained in dimensions of space and time alone, through the Stark and Zemann effects.
} 
through a diaphragm with two slits, but does not pass either one of them." Such statements that go with the notion of indeterminacy are parts of the conceptual structure, which has been developed in the attempt to interpret the formalism in terms of generalized classical concepts and which has been regarded as an inherent feature of the theory. However, the complete break with causality leads to a formalism that speaks for itself and disposes altogether of notions associated with indeterminate variables.

\subsection{Fortuitousness. Completeness}

In usual quantum mechanics, fortuitousness is not a primary concept, but enters the theory through the interpretation of the formalism in terms of probabilities for measurements. The insistence on the indeterminate particle as responsible for the click makes it impossible to see the genuine character of fortuitousness and, hence, the basic principle for quantum mechanics itself.

The lack of a rationale for the probabilistic character of usual quantum mechanics has given rise to doubts as to the completeness of the theory, which grew out of classical mechanics by quantization. The principle excludes the possibility of underlying causes, and probability now enters, as a primary concept, in the embodiment of symmetry in the distributions of clicks with assigned proper values. Thus, probability originates from the completely lawless side, rather than from the deterministic side, as in usual quantum mechanics.

\subsection{Measurement}

In usual quantum mechanics, the click is interpreted as a measurement of some variable of the particle. However, as is well-known, the notion of a measurement, which is taken over from classical physics, lacks a clear content, when applied to an indeterminate variable. Thus, the interpretation implies that the result of a measurement of an indeterminate variable does not establish a value that the variable had, but the click is taken to yield a value, with which the variable occurred, fortuitously, during the measurement process. For example, for a wave function heading towards two counters, the particle with indeterminate position variable is said to cause a click in one of the counters, yet the particle does not enter the counter that clicks. ${ }^{10}$ The tacit acceptance of this conceptual framework enforced by the notion of a cause for the fortuitous click has obscured the

${ }^{10}$ The question of the development of decoherence between the two components due to entangling with detector constituents, or the environment, has no bearing on this issue. 
recognition that something takes place that has not been grasped by the theory. The missing element is the discontinuity in the onset, which is unanalyzable. Thus the uncaused click bears no relation to a measurement.

\subsection{State of Object. Correlations}

The notion of a particle as an object in space (a thing) implies a state for the particle. Thus, in usual quantum mechanics, the wave function is seen as the state of the particle, and it has been an issue to reconcile this view with the interpretation of the wave function in terms of probabilities.

Since the theory of uncaused clicks does not deal with the behaviour of things, the entire formalism is from the outset recognized as symbolic, exclusively describing distributions of clicks in terms of the click vector. Thus, the notion of a wave function or state of a thing is eliminated (see Sec. 4).

The recognition of the symbolic character of the formalism, with no state of an object, clarifies issues concerning correlated clicks for two-particle wave functions that have continued to play an important role in the discussions of usual quantum mechanics. In this framework, the correlation implies that the observation of a click for one particle instantaneously endows the other particle with a state that depends on the chosen observation of the first particle. This conceptual problem is not present in the theory of no things in which there is no object to be assigned a state, and in which the correlation refers to a single discontinuity occurring at two different locations (see Sec. 3.4).

\subsection{Particle-Wave Duality. Planck's Constant}

In the whole development of quantal physics, Planck's constant has been considered as the symbol for the radical break with classical physics. In particular, Planck's constant was seen as a key to a synthesis of the particle and wave features of the observations connecting the momentum of the particle with a wave number. Particle-wave duality was later seen as part of a more general relation between particle variables and the generators of coordinate transformations, with Planck's constant as a proportionality factor.

The irrelevance of Planck's constant for quantum mechanics reveals the illusion in the idea of a particle-wave duality as well as of the enigmatic connection between particle variables and coordinate transformations. These concepts crept into ususal quantum mechanics together with Planck's constant by the felt need for a particle as a cause for the click. 
The intuitive feeling of the necessity of particles as cause for the clicks may be compared with the adherence to the ether. This medium appeared necessary as a carrier of the electromagnetic waves, but obscured the discovery of the principle of relativity.

\section{CONCLUDING REMARKS}

Genuine fortuitousness with its two faces, lack of cause and discontinuity, is found to be the principle that underlies quantum mechanics. The acceptance of the completely uncaused clicks goes against deeply held beliefs, but is consistent with all evidence and provides a simple principle for the theory.

\section{APPENDIX. EMBODIMENT OF SYMMETRY (SEE ALSO REF. 5)}

A group is defined in terms of the multiplication table that tells how the product of two elements leads to a third. The multiplication table can be solved in terms of matrices that thereby represent the group. In special cases, the matrices reduce to a number (one-dimensional representation), but if the group is non-Abelian, i.e., contains elements that do not commute, it has multidimensional matrix representations that are irreducible. The irreducibility implies that the proper values are inextricably interwoven so that it is not possible to divide the proper values for each matrix into two subsets, each of which transforms within itself and thus gives a representation. The irreducible representations of a symmetry group are the building blocks in terms of which symmetry expresses itself in numbers. It is shown in the following, how the multiplication table manifests itself in additive constraints for the matrices in an irreducible representation and how the symmetry is embodied in the click distributions through these constraints.

In a symmetry group with elements $\mathscr{U}_{1}, \mathscr{U}_{2}, \ldots, \mathscr{U}_{g}$, the matrices in an irreducible representation of dimension $d$ satisfy the orthogonality relations

$$
\sum_{n=1}^{n=g}\left\langle k\left|\mathscr{U}_{n}\right| J\right\rangle\left\langle l\left|\mathscr{U}_{n}^{-1}\right| l\right\rangle=\frac{g}{d} \delta_{l \jmath} \delta_{k l} .
$$

The relation (1) is familiar for the $\mathscr{D}$-functions of the rotation group, with the sum replaced by an integration over the Euler angles. 
Multiplication of (1) with $\langle l|\mathscr{U}| \imath\rangle$ for any $\mathscr{U}$ and summation over $l, l$ leads to the relation

$$
\mathscr{U}=\frac{d}{g} \sum_{n=1}^{n=g} \mathscr{U}_{n} \operatorname{Tr}\left\{\mathscr{U} \mathscr{U}_{n}^{-1}\right\}
$$

which expresses the matrix $\mathscr{U}$ as a linear combination of all the matrices, with coefficients that are traces (characters). The additive constraints (2) for all the irreducible representations are equivalent to the multiplicative constraints between the elements of the group.

A distribution of proper values $u$ for any $\mathscr{U}$ is characterized by a probability distribution $p(u)$ with mean value

$$
\langle\mathscr{U}\rangle=\sum_{u} u p(u)
$$

summed over all the proper values of $\mathscr{U}$. The distribution $p(u)$ is completely specified by the mean values of $\mathscr{U}$ and its powers $\mathscr{U}^{r}$, $r=0,1,2, \ldots, d-1$, which are also elements of the group. The probability distributions for the different elements is, therefore, exhaustively described by the mean values $\langle\mathscr{U}\rangle$.

The embodiment of the symmetry in the distributions of clicks that come by themselves consists in carrying the mathematical constraints (2) for the symmetry matrices into the same set of constraints for the mean values,

$$
\langle\mathscr{U}\rangle=\frac{d}{g} \sum_{n=1}^{n=g}\left\langle\mathscr{U}_{n}\right\rangle \operatorname{Tr}\left\{\mathscr{U} \mathscr{U}_{n}^{-1}\right\}
$$

It follows that the mean values must be of the form

$$
\langle\mathscr{U}\rangle=\operatorname{Tr}\{\rho \mathscr{U}\}
$$

involving a matrix $\rho$, which itself is a sum over all the mean values,

$$
\rho \equiv \frac{d}{g} \sum_{n=1}^{n=g} \mathscr{U}_{n}^{-1}\left\langle\mathscr{U}_{n}\right\rangle .
$$

Conversely, it is a consequence of the linearity of (4) that these constraints are fulfilled for mean values of the form (5), for any matrix $\rho$. For a unitary matrix $\langle\mathscr{U}\rangle^{*}=\left\langle\mathscr{U}^{-1}\right\rangle$, and the matrix $\rho$ is thus hermitean. 
The matrix $\rho$ can be diagonalized, with proper values $w(\mathscr{C})$ and proper vectors $|\mathscr{C}\rangle$,

$$
\rho=\sum_{\mathscr{C}}|\mathscr{C}\rangle w(\mathscr{C})\langle\mathscr{C}|
$$

leading to the probabilities (see (3) and (5))

$$
\langle\mathscr{U}\rangle=\sum_{\mathscr{C}} w(\mathscr{C})\langle\mathscr{C}|\mathscr{U}| \mathscr{C}\rangle, \quad p(u)=\sum_{\mathscr{C}} w(\mathscr{C})|\langle u \mid \mathscr{C}\rangle|^{2}, \quad|\mathscr{C}\rangle=\sum_{u}|u\rangle\langle u \mid \mathscr{C}\rangle
$$

in the basis $|u\rangle$.

A click assigned the proper value $u$ can be incorporated into any of the proper value distributions $|\langle u \mid \mathscr{C}\rangle|^{2}$. The weights $w(\mathscr{C})$ in (8) express this incompleteness in the experimental set-up, which implies that the total distribution decomposes into a sum of unconnected distributions.

\section{ACKNOWLEDGMENTS}

We would like to acknowledge stimulating discussions with Kurt Gottfried, Jørgen Kalckar, and Wladek J. Swiatecki. Valued comment came also from discussions with colleagues at the Weizmann Institute and the Hebrew University. Critical comments from the referees provided valuable inducements to clarify the argumentation.

\section{REFERENCES}

1. O. Ulfbeck and A. Bohr, Found. Phys. 31, 757 (2001).

2. J. A. Wheeler and W. H. Zurek, eds. Quantum Theory and Measurement (Princeton University Press, Princeton, NJ, 1983). We have been informed that a new edition is in preparation.

3. D. F. Styer et al., "Nine formulations of quantum mechanics," Amer. J. Phys. 70, 288 (2002).

4. S. Goldstein, “Quantum theory without observers," Phys. Today 51, 42 (March 1998) and Phys. Today 51, 38 (April 1998). See also the exchanges of opinion on these articles in Phys. Today 52, 11, 89 (February 1999).

5. A. Bohr and O. Ulfbeck, Rev. Mod. Phys. 67, 1 (1995).

6. G. Kaiser, J. Math. Phys. 22, 705 (1981). 